\title{
Statistical design and analysis in trials of proportionate interventions: a systematic review
}

\author{
Jane Candlish $^{1 *}$ (D), M. Dawn Teare ${ }^{1}$, Judith Cohen ${ }^{1,3}$ and Tracey Bywater ${ }^{2}$
}

\begin{abstract}
Background: In proportionate or adaptive interventions, the dose or intensity can be adjusted based on individual need at predefined decision stages during the delivery of the intervention. The development of such interventions may require an evaluation of the effectiveness of the individual stages in addition to the whole intervention. However, evaluating individual stages of an intervention has various challenges, particularly the statistical design and analysis. This review aimed to identify the use of trials of proportionate interventions and how they are being designed and analysed in current practice.
\end{abstract}

Methods: We searched MEDLINE, Web of Science and PsycINFO for articles published between 2010 and 2015 inclusive. We considered trials of proportionate interventions in all fields of research. For each trial, its aims, design and analysis were extracted. The data synthesis was conducted using summary statistics and a narrative format.

Results: Our review identified 44 proportionate intervention trials, comprising 28 trial results, 13 protocols and three secondary analyses. These were mostly described as stepped care $(n=37)$ and mainly focussed on mental health research $(n=30)$. The other studies were aimed at finding an optimal adaptive treatment strategy $(n=7)$ in a variety of therapeutic areas. Further terminology used included adaptive intervention, staged intervention, sequentially multiple assignment trial or a two-phase design. The median number of decision stages in the interventions was two and only one study explicitly evaluated the effect of the individual stages.

Conclusions: Trials of proportionate staged interventions are being used predominantly within the mental health field. However, few studies consider the different stages of the interventions, either at the design or the analysis phase, and how they may interact with one another. There is a need for further guidance on the design, analyses and reporting across trials of proportionate interventions.

Trial registration: Prospero, CRD42016033781. Registered on 2 February 2016.

Keywords: Systematic review, Complex intervention, Trial, Proportionate intervention, Stepped care, Adaptive treatment strategy, Adaptive intervention, Sequential multiple assignment randomised trial, Proportionate universalism

\section{Background}

Many health, educational and social interventions have multiple components. For a proportionate intervention, these complex multi-component interventions are delivered in a proportionate or adaptive manner, in which the components of the intervention are delivered in response

*Correspondence: jane.candlish@sheffield.ac.uk

${ }^{1}$ ScHARR, University of Sheffield, 30 Regent Court, S1 4DA Sheffield, UK

Full list of author information is available at the end of the article to an individual's need over time. Other analogous terms for a proportionate intervention include adaptive intervention, dynamic treatment regime and stepped care.

Proportionate interventions are multi-stage and defined by a series of decision rules. The key features are critical time points, tailoring variables and treatment options. An example of an adaptive intervention was described by Almirall et al. [1]. This intervention aimed to improve outcomes for children with autism who had minimal verbal 
skills. The treatment was discrete teaching and was delivered at stage 1 as two sessions per week over 12 weeks. At 12 weeks, the child was assessed for change in spontaneous communication utterances since baseline (the tailoring variable). At the critical time point at 12 weeks, if the spontaneous communication utterances increased by at least $25 \%$, then the discrete teaching treatment continued at two sessions per week for the next 12 weeks. If the change was below this, then the discrete teaching treatment increased to three sessions per week.

The proportionate approach is based on the notion that individuals differ in their response to treatment. Individuals who require a step-up, step-down or switch in intervention receive it. For those who are responding to the current intervention, there is no increase in burden, such as side effects or invested time. Additionally, all interventions incur costs and multi-component interventions can be both costly and resource intensive. Providing treatment appropriate to individual need should improve efficiency by reducing the costs of unnecessary further treatments whilst conserving resources for those in greatest need. Proportionate interventions are in keeping with recommendations from the Strategic Review of Health Inequalities in England after the 2010 Marmot Review [2], which stated that actions and interventions should be both universal and targeted to reflect the level of need or disadvantage. This idea was termed 'proportionate universalism'. Recent advances include just-in-time adaptive interventions, which deliver treatment sensitive to an individual's changing needs for support. With technology, treatment can be based on measurements of rapidly changing factors. In health or behavioural change interventions, this allows a treatment to be delivered when a person is (a) vulnerable or open to positive changes and (b) receptive. Nahum-Shani et al. [3] developed a conceptual framework to help guide the development of just-in-time adaptive interventions, which is likely to be used more commonly in clinical trials in the near future.

Evaluating a proportionate intervention in a randomised controlled trial presents fresh challenges for the statistical study design and analysis outside current guidelines for complex intervention research $[4,5]$. Teams developing such proportionate interventions may wish to optimise the intervention and thus, may want to evaluate the incremental effectiveness of the individual stages in addition to the overall intervention. In general, trials randomise individuals or clusters to a whole intervention package to assess effectiveness. However, a proportionate intervention creates a variable number of levels of intervention and frequently multiple hierarchical levels of clustering occur, each dependent upon outcomes at the previous stage of intervention. Clustering may be non-random and dependent on an intermediate outcome.
This review aimed to identify trials of proportionate interventions and how they are being designed and analysed in current practice. Research into proportionate and adaptive interventions has previously been done in other forms or with a slightly different focus to this review $[3,6]$. Early work by Collins et al. [6] presents a conceptual framework for adaptive interventions. They discuss key design principles including choice of tailoring variables and the derivation of good decision rules. A good decision rule is objective and comprehensive and it will ensure intervention components are delivered to individuals at the intended intensity. Nahum et al. [3] reviewed how adaptive interventions use decision rules to link individual responses with intervention options and the repeated use of these rules to adapt interventions over time in response to the changing response of individuals. They discuss how sequential multiple-assignment randomised trials (SMARTs) can be used to construct adaptive interventions, using a case study of an adaptive intervention for children with attention deficit hyperactivity disorder (ADHD) to illustrate analysis methods for the SMART design. They compare first- and second-stage intervention options, and the interventions embedded within the SMART design. Proportionate or adaptive interventions are desirable due to the heterogeneous responses to treatments. Some people may need only a low-intensity intervention while others may need a higher intensity or an alternative intervention. However, the design of adaptive intervention strategies must be driven by the scientific research question. Almirall et al. [1] present an informative review of the optimal design and evaluation of adaptive interventions in education research.

The current review moves beyond the work that has already been conducted. It systematically reviews the methods used in studies of proportionate interventions. It will be useful for those planning and analysing trials in this area, since we present fields in which proportionate interventions are currently being utilised, the types of tailoring variables and the decision rules used.

We conducted a systematic review of published trials to present the types of proportionate interventions being evaluated and the design and analysis methods being undertaken in current practice. Without knowing the variety of proportionate interventions and scenarios that exist, methodological work investigating suitable design and analysis strategies cannot be focused appropriately. The specific objectives of this systematic review were to:

1 Explore how trials of proportionate interventions are being designed in practice

2 Review the type of statistical design and analysis methods being implemented in trials involving staged proportionate interventions 
3 Review whether trials of proportionate interventions are being analysed differently to trials of non-proportional interventions and if the component parts are considered in the analysis.

\section{Methods}

Details of the protocol for this systematic review were registered on PROSPERO (CRD42016033781). We conducted the review according to the Preferred Reporting Items for Systematic Reviews and Meta-Analyses (PRISMA) guidelines for reporting systematic reviews where relevant [7]. A completed PRISMA checklist [7] is available as Additional file 1.

\section{Literature search}

Proportionate interventions evaluated in a randomised controlled trial between 2010 and 2015 inclusive were sought. Electronic searches were undertaken using the databases: MEDLINE (OvidSP), Web of Science (Core Collection) and PsycINFO. The search terms were any of the following in the title or abstract: 'proportionate universalism,' 'proportionate intervention', 'proportionate treatment', 'staged intervention', 'staged treatment', 'adaptive treatment regimen, 'adaptive intervention, 'adaptive treatment strategy', 'dynamic treatment regimen', 'multilevel intervention' or 'stepped care.' The search strategy was based on the Cochrane Highly Sensitive Search Strategies for identifying randomised trials [8]. The start date of the 6-year time frame was chosen based on the 2010 publication date of the Marmot review [2], which referred to proportionate universalism. We anticipated no trials would use the term 'proportionate universalism' prior to this review and anticipated an increase in the use of such interventions post publication of the Marmot Review [2]. The final search was conducted in March 2016 (after piloting and refining the search strategy). Search strategies were developed that were relevant to the database requirements (see Fig. 1 for the MEDLINE search strategy). The intention of the systematic review was to provide a thorough overview of the types of trials of proportionate interventions being used in practice but not to be exhaustive; therefore, additional hand searching or searching of clinical trial registers was not incorporated.

\section{Eligibility criteria}

All search results that were trials or pilot studies (including protocols) that evaluated interventions delivered proportionate to need were eligible. An intervention proportionate to need was defined as one in which there is a variation in the intervention dependent upon either an intermediate or primary outcome measured prior to the study endpoint. There should be decision stages and at each stage there should be treatment options based on tailoring variables and predefined decision rules. Interventions that are tailored without decision rules were excluded from this review. We excluded observational studies and those not in English. Where more than one article for a single study was found, the main article of published results was included if available and if it superseded any protocol or cost-effectiveness study. We considered all therapeutic areas and imposed no restrictions on the participants.

\section{Quality control}

We did not undertake a quality assessment of the identified studies as the purpose of this review was to understand what interventions and trial designs are being

1. (randomi\#ed controlled trial OR controlled clinical trial).pt. OR randomi\#ed.ab. OR placebo.ab. OR clinical trial as topic.sh. OR randomly.ab. OR trial.ti.

2. limit 1 to $\mathrm{yr}=" 2010-2015 "$

3. (proportionate universalism OR proportionate intervention\$ OR proportionate treatment $\$$ or staged intervention $\$$ or staged treatment $\$$ OR multilevel intervention\$ OR multi-level program\$ OR multi-level system\$ OR multi-level treatment $\$$ OR stepped care).ab,ti.

4. (adaptive treatment\$ OR adaptive treatment regime\$ OR adaptive intervention\$ OR adaptive treatment strateg\$ OR dynamic treatment regime\$).ab,ti.

5. 3 OR 4

6. 1 AND 5

7. 2 AND 6

8. limit 7 to English language

Fig. 1 MEDLINE search strategy 
used in practice and how they are being designed and analysed.

\section{Study selection}

Study selection based on the eligibility criteria was performed by review author JCa, who identified relevant results. All duplicates were removed. At the initial screening stage, titles and abstracts were assessed to identify if the study was eligible. The full articles of studies meeting the review criteria were obtained and inspected to identify relevant studies that fulfil the inclusion criteria. Two second authors (JCo and MDT) reviewed a random sample of ten results each to assess agreement and the clarity of eligibility criteria.

\section{Data extraction and synthesis}

A data extraction tool was developed for this review in an Excel spreadsheet. The data extraction tool was piloted by reviewers JCa, JCo and MDT and refined based on feedback. This review evaluated designs and methods used in proportionate intervention trials; therefore, a metaanalysis was not appropriate. We collected the following information with the data extraction tool: publication year, location of study (country), therapeutic area, type of study (trial results, protocol or secondary analysis), design type, aim, eligibility criteria, intervention, tailoring variable, decision rules, number of decision stages, control intervention, final study follow-up period, sample size, primary outcome, overall statistical model, and whether an analysis of different stages was undertaken.

The review results were presented using summary statistics. A narrative synthesis describes any similarities and differences among the included studies. We grouped studies by design type, and study characteristics were tabulated to allow a comparison of the main features.

\section{Results}

\section{Study selection}

Figure 2 presents the process of study selection in this systematic review. Of the 531 unique records identified from the database search, we identified 44 eligible studies (all papers included are listed in Additional file 2). These 44 studies consisted of 28 trial results, 13 protocols and three secondary analyses. The narrative syntheses have been split into two subcategories by type of study design, stepped care and optimal treatment strategy. Inclusion of a control arm was not required for eligibility. Due to the nature of assessing proportionate interventions, some results did not include a control arm, either due to ethical arguments or because the objective was to identify an optimal treatment strategy.

\section{Study characteristics}

Table 1 presents an overview of the included studies. There were 18 studies based in the United States, 14 in the Netherlands, one in the Netherlands and Belgium, two in each of Australia, England and Scotland, Norway and Sweden, and three based in other countries (India, Nigeria and a multi-site study in France, Hungary, Romania and Slovakia).

The median number of decision stages (points at which the intervention was adapted according to need based on predefined decision rules) was 2 (interquartile range 1 to 3 ). The median length of trial follow-up was 12 months (interquartile range 6 to 12 months) and the median sample size was 236 (interquartile range 150 to 387).

\section{Stepped care}

Table 2 is a summary of the included stepped-care studies. A total of $84 \%$ (37 of 44) of the studies followed a steppedcare model for the intervention. The stepped-care model is recommended by the National Institute for Health and Care Excellence (NICE) [9] for the provision of services for common mental health disorders. In a stepped-care model, the least intensive intervention (or the lowest level of intervention) is delivered first to all patients, and patients step up or down the stepped-care treatment pathway dependent upon their response to the previous intervention step.

Figure 3 represents the flow of patients through an example of a typical stepped-care trial with three treatment steps. The key principles of stepped care are to provide the most appropriate and best treatment according to need, to reduce the burden on patients by providing only the treatment required and to improve cost-effectiveness by providing the level of intervention required for a positive outcome [9]. The reduction of costs for those who respond to lower-intensity interventions can free up resources for those who require more intensive treatment [10].

The majority of stepped-care studies (73\%; 27 of 37) focussed on the therapeutic areas of depression, anxiety, stress or some form of mental health disorder. Other therapeutic areas targeted included: weight loss $[11,12]$, alcohol consumption [13, 14], eating disorders [15, 16], whiplash injuries [17], blood pressure control [18], resilience and well-being of families living with childhood chronic illness [19], and impairment in older dizzy people [20].

The interventions often involved some form of watchful waiting period for the first step followed by regular monitoring at predefined follow-up times of an outcome measure (either secondary or primary). Based on this outcome measure, decisions were made whether to progress to the next step or not. This process continued for however many steps were included in the intervention. Based on the individual's outcome, at each decision stage the options for the following step were often: (1) a choice of treatments, (2) continue with the same treatment, (3) augment the treatment or (4) discontinue the treatment 


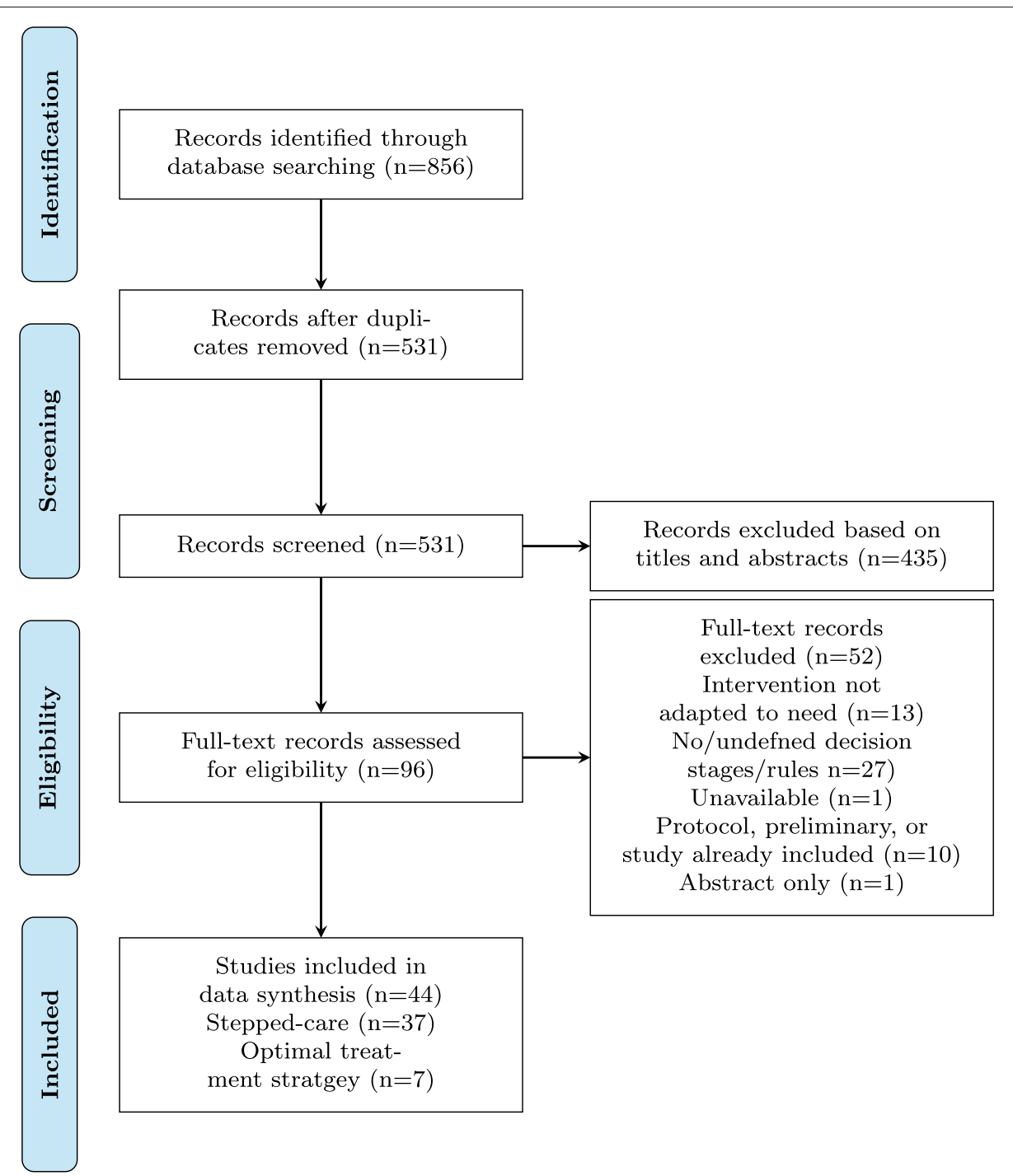

Fig. 2 PRISMA study flow diagram. Number of records identified, included and excluded during the literature search

altogether. The progression of treatment steps for interventions aimed at mental health disorders commonly included a watchful waiting period, bibliotherapy, guided self-help, or psychotherapy sessions (either individual or group based), with a possible progression to medication (for example, antidepressants).

Control conditions were generally usual care or enhanced usual care with others being assessment only [19], waiting list control [21] or the active treatment delivered in a non-stepped model [12, 22, 23]. Four of the stepped-care trials had no explicit control [18, 24-26]. In one study, it was argued that the lack of control was partially inherent to the stepped-care design since it was unethical to assign individuals to a waiting list control after the first treatment step if they needed further treatment [26].
Measures were taken at baseline, at each decision stage and after the end of the final intervention stage. Generally, follow-up measures were also taken a number of months after completion of the interventions to assess if the effects were sustained. Our search results included both individually randomised and cluster-randomised trials.

A variety of statistical analysis methods were used, dependent upon the outcome measures and main aims. Longitudinal data were incorporated into many of the analyses. Mixed-effects models, containing both fixed and random effects, were used as the statistical analysis method in $38 \%$ of studies (14 of 37; Table 2). They were used to account for both longitudinal data and the clustering effects of National Health Service (NHS) trusts, therapists and other health professionals. Repeated measures analysis of variance (ANOVA) was used in three studies 
Table 1 Overview of studies included in the systematic review

\begin{tabular}{|c|c|c|c|c|c|}
\hline First author & Date $^{a}$ & Therapeutic area & Country & Follow-up $p^{b}$ & $N^{c}$ \\
\hline Ell [54] & 2010 & Depression and anxiety & United States & 12 & 387 \\
\hline $\begin{array}{l}\text { Van't } \\
\text { Veer-Tazelaar [55] }\end{array}$ & 2010 & Depression and anxiety & The Netherlands & 12 & 170 \\
\hline Braamse [56] & 2010 & $\begin{array}{l}\text { Distress after autologous stem cell } \\
\text { transplantation }\end{array}$ & The Netherlands & 10 & 286 \\
\hline Patel [57] & 2010 & Depression and anxiety & India & 12 & 2796 \\
\hline Gilliam [25] & 2010 & Obsessive-compulsive disorder & United States & 3 & 14 \\
\hline Kay-Lambkin [58] & 2010 & $\begin{array}{l}\text { Depression among methamphetamine } \\
\text { users }\end{array}$ & Australia & 5 & 8 \\
\hline Richter [18] & 2011 & Blood pressure & France, Hungary, Romania, Slovakia & 6 & 256 \\
\hline Weiss $^{d}[32]$ & 2011 & Prescription opioid dependence & United States & 6 & 653 \\
\hline Mitchell [16] & 2011 & Bulimia nervosa & United States & 12 & 293 \\
\hline Seekles [59] & 2011 & Depression and anxiety & The Netherlands & 6 & 120 \\
\hline Tolin [23] & 2011 & Obsessive-compulsive disorder & United States & 3 & 34 \\
\hline $\begin{array}{l}\text { van der Leeden } \\
{[26]}\end{array}$ & 2011 & Anxiety in children & The Netherlands & 6 & 133 \\
\hline Apil [60] & 2012 & Depression & The Netherlands & 12 & 136 \\
\hline Karp [61] & 2012 & Depression and chronic pain & United States & 12 & 250 \\
\hline Shortreed ${ }^{d}[33]$ & 2012 & Schizophrenia & United States & 18 & 1460 \\
\hline Dozeman [62] & 2012 & Depression and anxiety & The Netherlands & 10 & 185 \\
\hline Nordin [24] & 2012 & Stress management of cancer patients & Sweden & 12 & 300 \\
\hline Jakicic [11] & 2012 & Weight loss & United States & 18 & 363 \\
\hline Wang $^{d}[34]$ & 2012 & Oncology & United States & 7 & 150 \\
\hline Pommer [63] & 2012 & $\begin{array}{l}\text { Depression and anxiety in patients with } \\
\text { asthma or COPD }\end{array}$ & The Netherlands & 24 & 160 \\
\hline Lamb [17] & 2012 & Whiplash injuries & England and Scotland & 12 & 3851 \\
\hline Krebber [27] & 2012 & $\begin{array}{l}\text { Distress in head and neck and lung cancer } \\
\text { patients }\end{array}$ & The Netherlands & 12 & 176 \\
\hline Borsari [21] & 2012 & Alcohol consumption & United States & 9 & 598 \\
\hline $\operatorname{Rose}^{d}[30]$ & 2013 & Smoking cessation & United States & 6 & 606 \\
\hline Watson [13] & 2013 & Alcohol consumption & England and Scotland & 12 & 529 \\
\hline Oosterbaan [29] & 2013 & Common mental disorders & The Netherlands & 8 & 163 \\
\hline van Dijk [64] & 2013 & $\begin{array}{l}\text { Depression among patients with diabetes } \\
\text { and/or coronary heart disease }\end{array}$ & The Netherlands & 12 & 236 \\
\hline Arving [65] & 2013 & Stress management of cancer patients & Norway & 24 & 300 \\
\hline Mattsson [28] & 2013 & Depression and anxiety & Sweden & 24 & 200 \\
\hline Carels [12] & 2013 & Weight loss & United States & 4 & 52 \\
\hline van der Aa [66] & 2013 & Depression and anxiety & The Netherlands and Belgium & 24 & 230 \\
\hline Kasarid $^{d}[31]$ & 2014 & $\begin{array}{l}\text { Communication for minimally verbal } \\
\text { children with autism }\end{array}$ & United States & 8 & 61 \\
\hline Muntingh [67] & 2014 & Panic and anxiety & The Netherlands & 12 & 180 \\
\hline Kilbourne $^{d}[36]$ & 2014 & Mood disorder & United States & 24 & 1600 \\
\hline Hamall [19] & 2014 & Families living with childhood chronic illness & Australia & 6 & 1050 \\
\hline Gureje [68] & 2015 & Depression & Nigeria & 12 & 1190 \\
\hline Stoop [69] & 2015 & $\begin{array}{l}\text { Depression and anxiety in patients with } \\
\text { diabetes, asthma or COPD }\end{array}$ & The Netherlands & 18 & 46 \\
\hline Stam [20] & 2015 & Impairment in older dizzy people & The Netherlands & 12 & 300 \\
\hline
\end{tabular}


Table 1 Overview of studies included in the systematic review (Continued)

\begin{tabular}{|c|c|c|c|c|c|}
\hline First author & Date $^{a}$ & Therapeutic area & Country & Follow-up $b$ & $N^{c}$ \\
\hline Lock [15] & 2015 & Anorexia nervosa & United States & 6 & 45 \\
\hline $\begin{array}{l}\text { Schuurhuizen } \\
{[70]}\end{array}$ & 2015 & $\begin{array}{l}\text { Distress in patients with metastatic } \\
\text { colorectal cancer }\end{array}$ & The Netherlands & 11 & 715 \\
\hline Haug [71] & 2015 & Panic and anxiety & Norway & 12 & 173 \\
\hline Salloum [72] & 2015 & Post-traumatic stress in children & United States & 3 & 53 \\
\hline$W_{u^{d}}[35]$ & 2015 & Bipolar disorder & United States & 3 & 365 \\
\hline Painter [73] & 2015 & Depression in HIV patients & United States & 12 & 245 \\
\hline
\end{tabular}

COPD chronic obstructive pulmonary disease

apublication date

bFinal follow-up post baseline in months

'Sample size

dOptimal-treatment strategy subcategory

$[25,27,28]$; however, this method does not successfully deal with missing values. In contrast, mixed-effects models assume data are missing at random and they allow for imbalanced or missing observations within patient.

Six stepped-care studies included or planned some form of analysis of the different stages. These included:

1. summaries of outcome measures presented per treatment step [18],

2. an analysis of outcomes after steps 1 and 2 [29],

3. an analysis at the end of each step and the end of the whole intervention as well as a comparison of differences in weight loss and self-monitoring characteristics between those who were stepped down and those who continued to receive treatment in the stepped-care arm [12],

4. a planned analysis of demographic data to compare the characteristics of those who agreed to participate in steps 2 and 3 compared to those who declined (for eligible patients) [19],

5. the percentages of children free of any anxiety disorder after each treatment phase and by intervention [26],

6. analysis of outcomes after step 1 and analysis of outcomes after step 2 adjusting for the intervention received in step 1 and any interactions between the step 1 and step 2 interventions [17].

The objectives of Lamb et al. [17] were to evaluate the effectiveness of step 1 , step 2, and the combined effects of the treatments together. This was made possible by designing two linked pragmatic randomised controlled trials. In step 1, emergency departments were cluster randomised to the Whiplash Book or usual care, and individual consent was not sought at this stage. In step 2, participants who received either of the step 1 treatments and were eligible after step 1 (persistent symptoms at 3 weeks) were individually randomised at step 2 to either one physiotherapist advice session or up to six physiotherapist advice sessions.

\section{Optimal adaptive treatment strategy}

Table 3 presents a summary of the studies of optimal adaptive treatment strategies. A total of $16 \%$ (7 of 44) of the review studies were aimed at finding an optimal treatment strategy. Treatments consist of more than one phase. Unlike most of the stepped-care studies, randomisation occurs more than once and there was often no true control, since the different adaptive treatment strategies were compared to one another. Six of the studies were explicitly defined as SMARTs, with the other study based on a two-phase trial design evaluating an adaptive smoking cessation treatment strategy [30].

The optimal treatment strategy studies included three with trial results [30-32], three secondary analyses of trials [33-35] and one trial protocol [36]. All seven studies were based in the United States. Therapeutic areas covered were oncology [34], schizophrenia [33], depression and anxiety [36], bipolar disorder [35], patients dependent on prescription opioids [32], smoking cessation [30] and communication for minimally verbal children [31].

Five of the optimal treatment strategy studies were based on two phases or stages of intervention and two studies used a three-phase design [34, 36]. A measurement at the end of each phase was used to assess the response and thus, progression to the next phase. Participants were generally randomised to phase 1 treatments. If they were classified as responders to phase 1 , they continued this treatment whereas non-responders were randomised to the following phase treatments. An example design is represented in Fig. 4. The number of treatments at each randomisation phase varied greatly between studies. In phase 1, there were between two and six treatments randomised (two treatments [31, 32, 36], three treatments [35], four treatments [34] or five treatments [33]). No control group was used in four of the studies [31, 33, 34, 36]. One study used a placebo in stage 1 [35] and usual care was used in another [32].

In general, more complex analysis methods were used for the optimal adaptive treatment strategies compared 


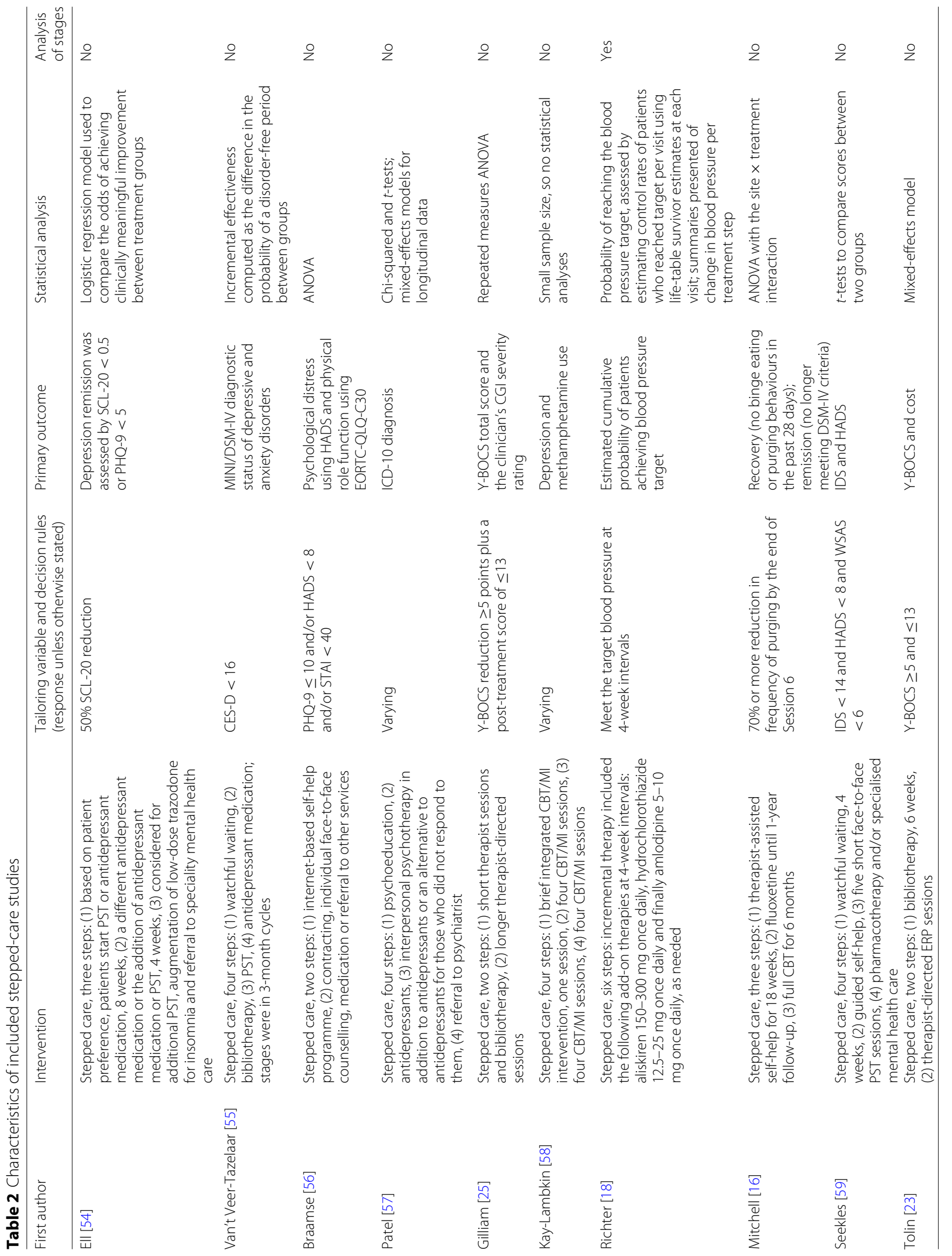




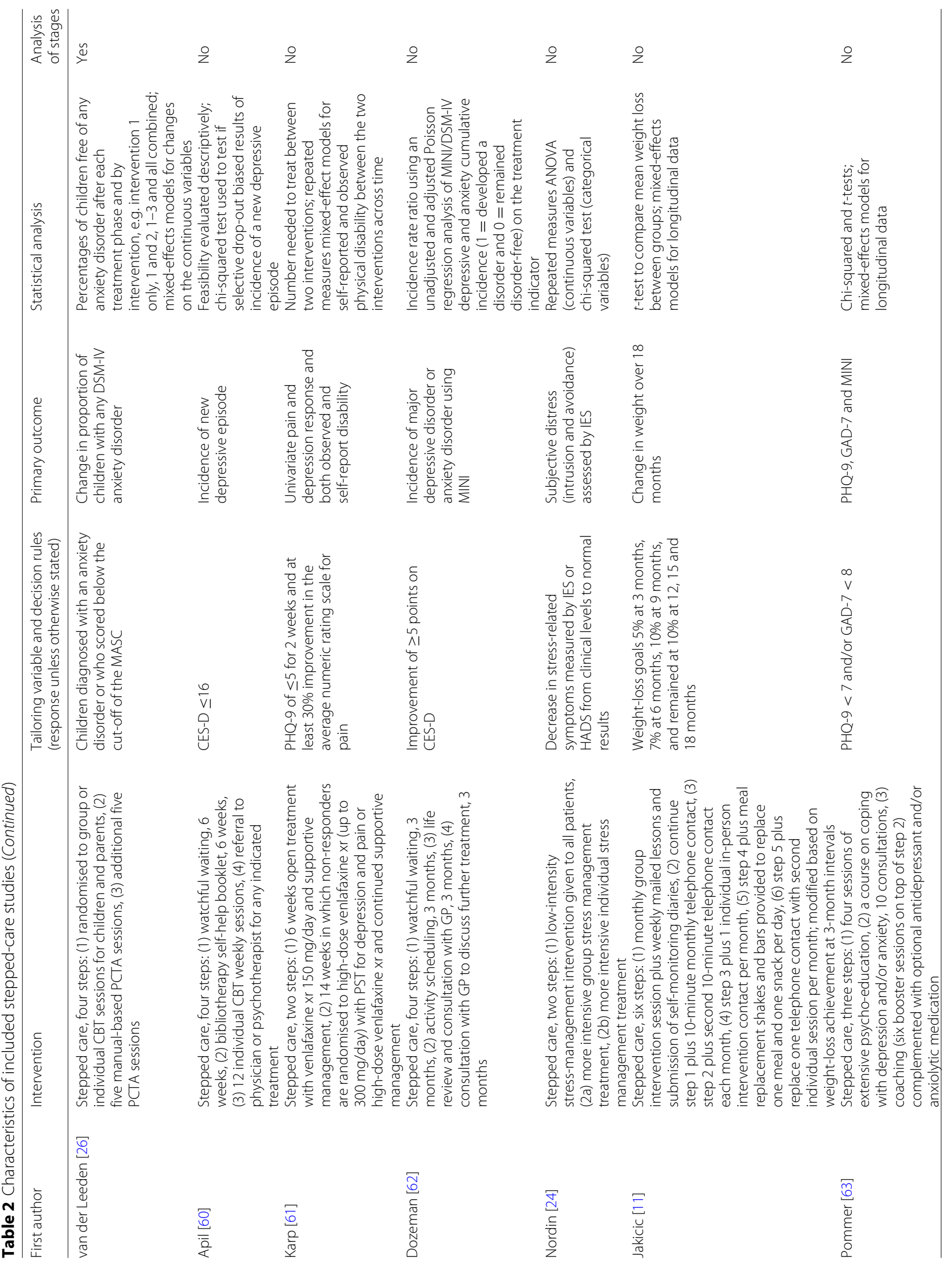




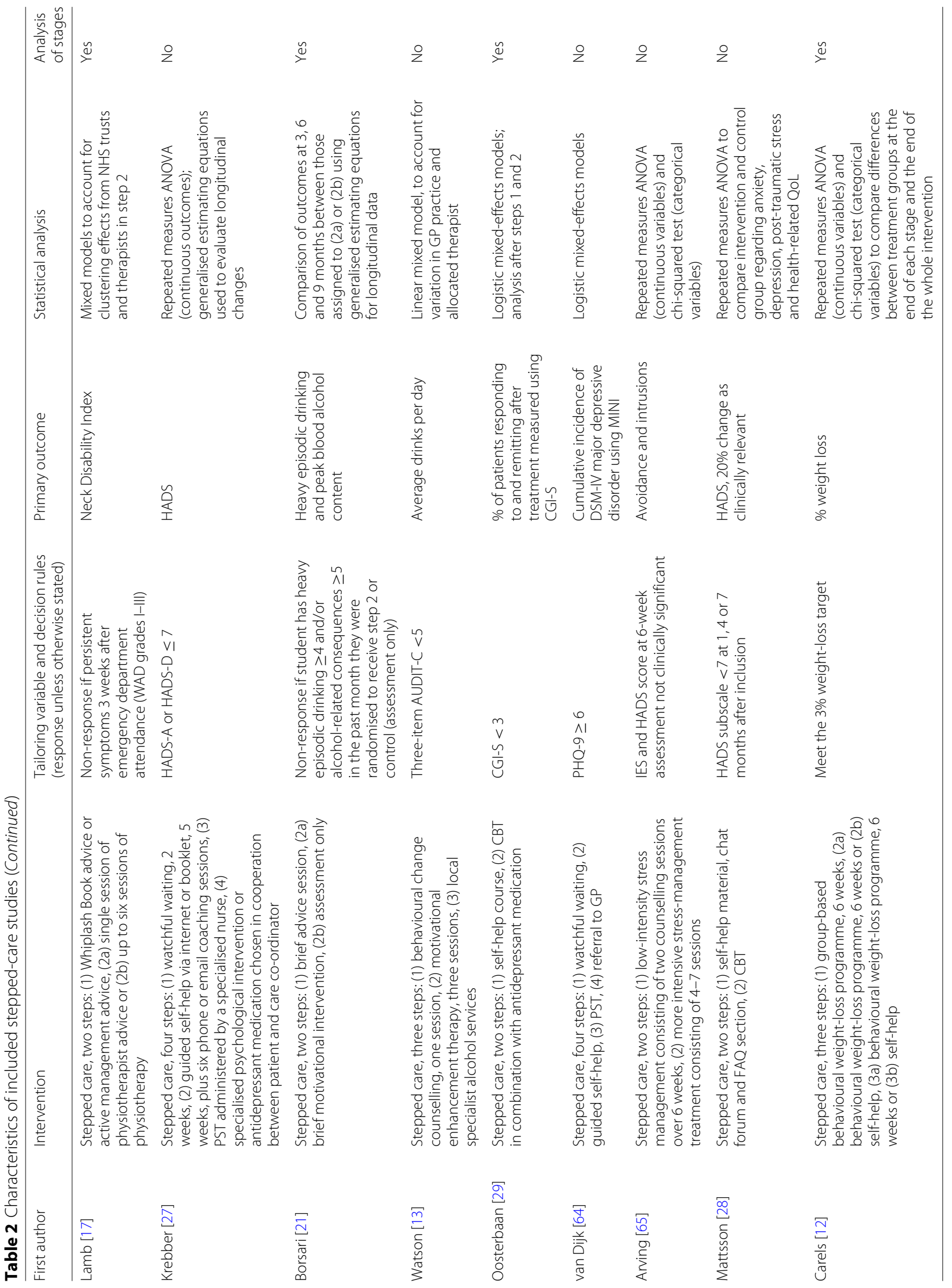




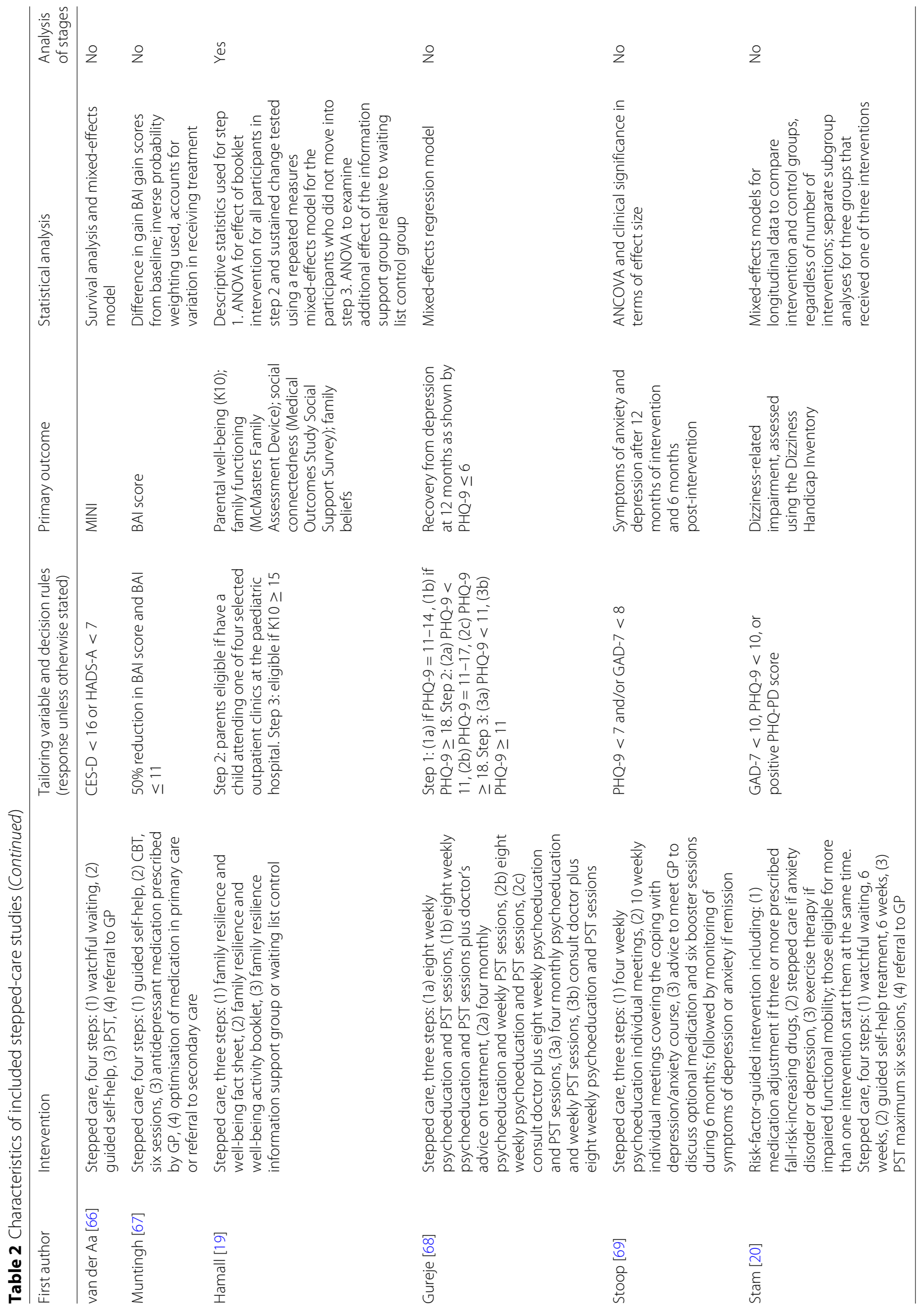




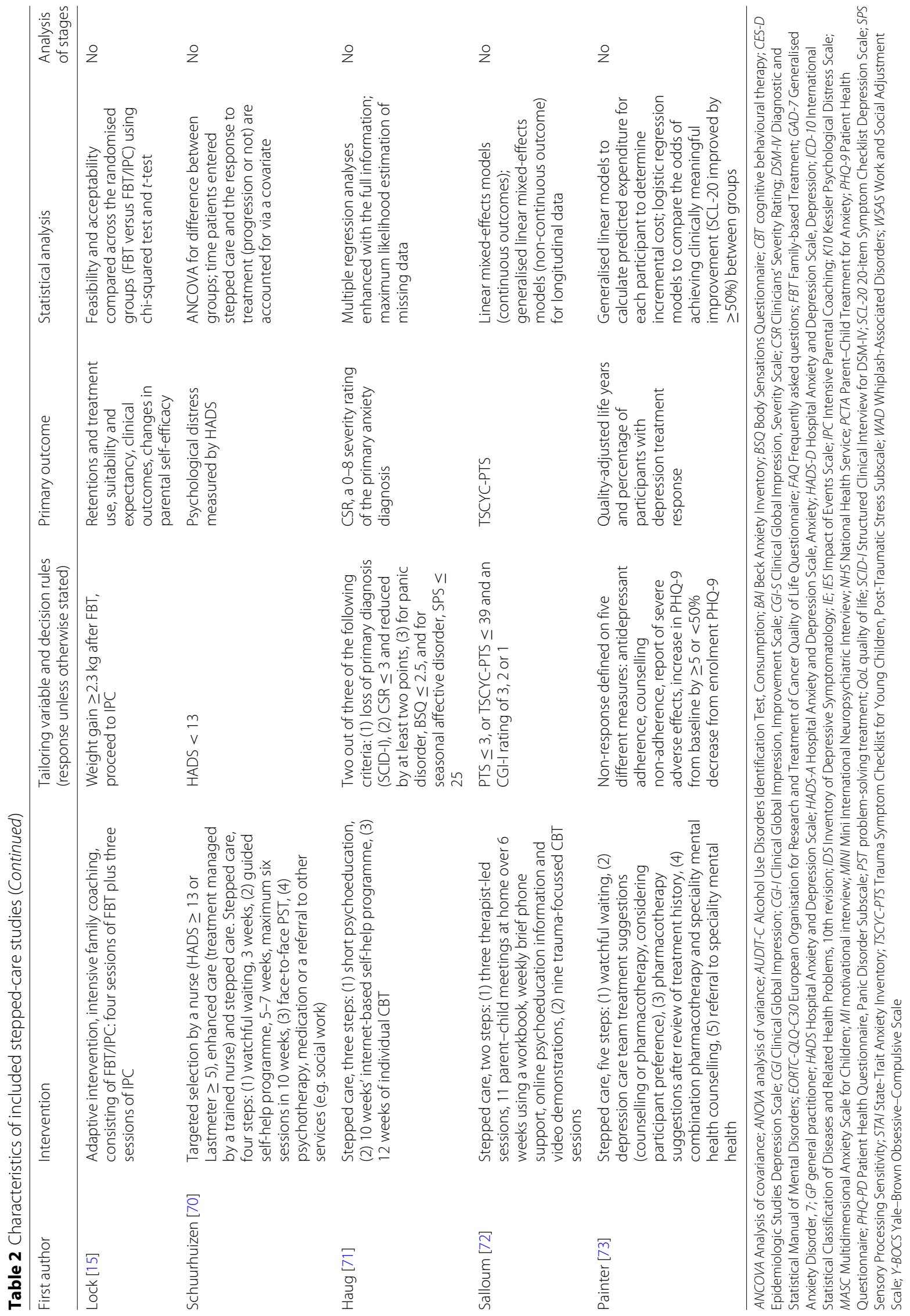




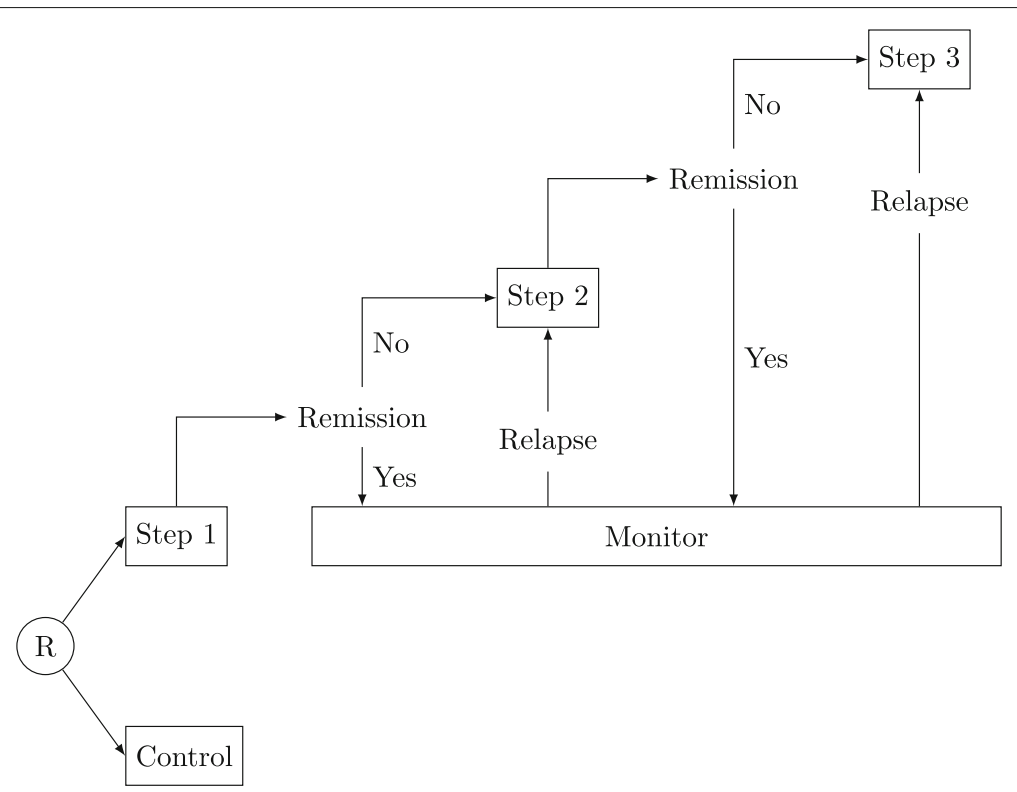

Fig. 3 Example of a stepped-care trial with three steps and the option to rejoin treatment if relapse occurs. R randomise

to the stepped-care trials. Inverse probability weighting methods were used to estimate the outcome means associated with each of the two-stage dynamic treatment regimes [34]. A comparison of two treatment conditions was done using the phase 2 endpoint and generalised estimating equations (to account for correlation among measurements of patients from the same site) [32]. Other methods to estimate the optimal treatment strategy included: Q-learning [35], marginal structural models [33] and mixed-effects models [31,36]. The studies were generally interested in estimating the optimal treatment strategy as a whole rather than considering the effects of each treatment stage. Different stages of the interventions were considered in some analyses, including measuring those who responded after stage 1 and randomising those who did not respond to stage 2 [32] and weighted regression to compare outcomes between the three embedded adaptive treatments, including an indicator for stage 1 and stage 2 treatments and accounting for the probability of a participant following their assigned sequence of treatments based on randomisation sequence [31].

\section{Discussion}

\section{Main findings}

The results suggest that trials are being designed in various therapeutic areas that fit the proportionate universal framework. Most results were conducted in developed countries. The term 'proportionate universalism' was not used within the identified studies. Other terminology used included: stepped care, adaptive treatment strategy, dynamic treatment regimen and SMART. In the review, eligible studies fell into two main subcategories of designs: trials using the stepped-care design (to provide treatment dependent on need) or aimed at identifying an optimal treatment strategy (when more than one treatment was available at various stages and administered dependent upon need). The stepped-care model begins with a lower level of intervention at the first step and treatment is administered at further steps only to those in need. Randomisation generally occurs only at baseline. The optimal treatment strategy trials inform decisions on how and when to alter treatment. They generally involved randomisation at each stage dependent upon the response at the end of the previous stage.

Mental health disorders were the most common therapeutic area of research in this review. This is most likely because a large majority of the results were stepped-care trials, which is a NICE-recommended pathway for mental health care [9]. Reasons for using a proportionate intervention were mainly based around costs and providing the level of care required by an individual. This is particularly relevant in mental health and complex interventions, since they are often fairly resource-intensive (both in time and costs).

The statistical methods used varied greatly based on the outcome measures, though longitudinal data are generally a feature of trials of a proportionate intervention. The trials need to update and measure the adjusting needs of patients during delivery of the intervention. ANOVA and repeated measures ANOVA were used in a number of analyses. However, these are not recommended as a general approach for longitudinal data due to the following limitations: (1) they 


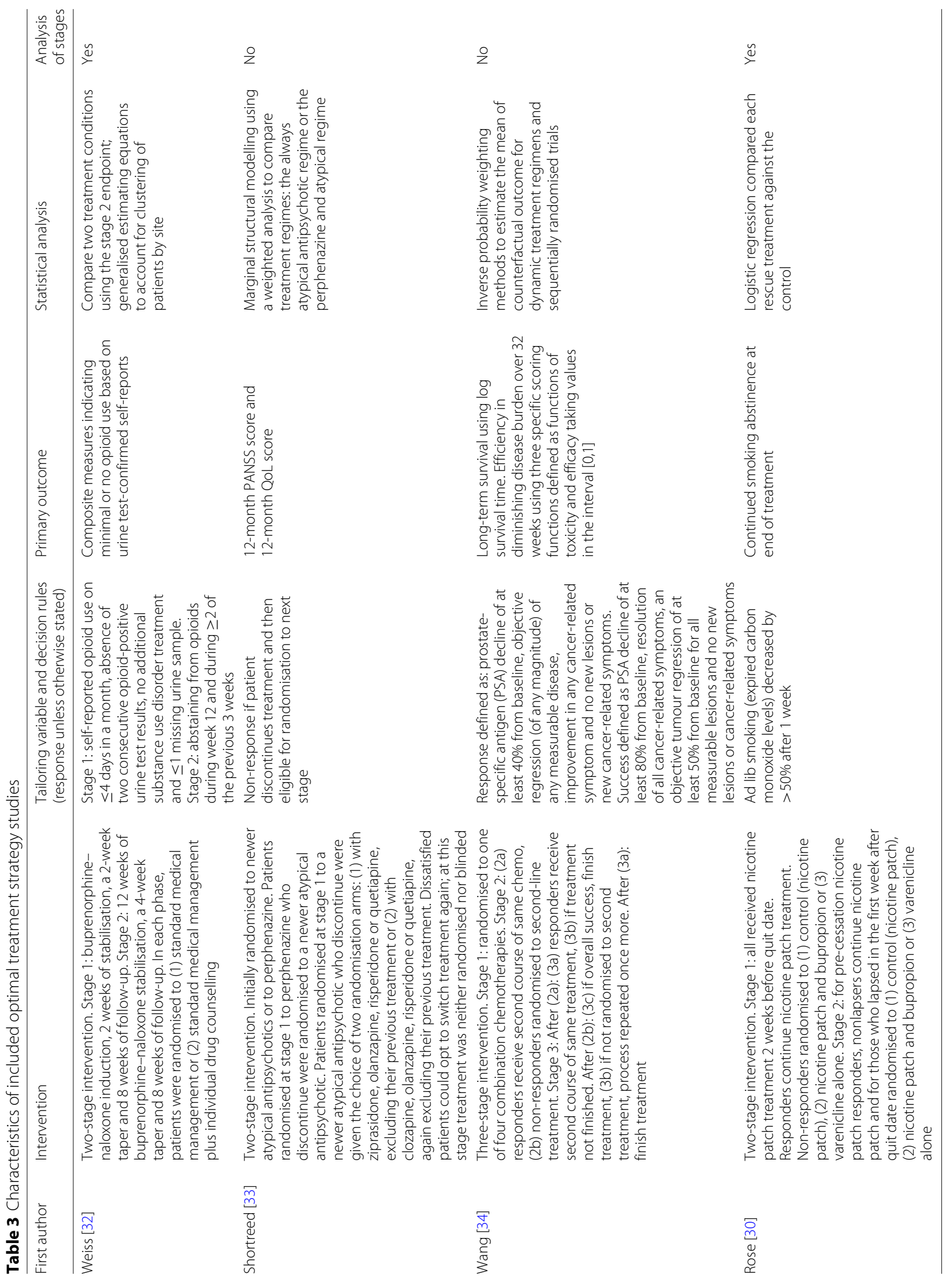




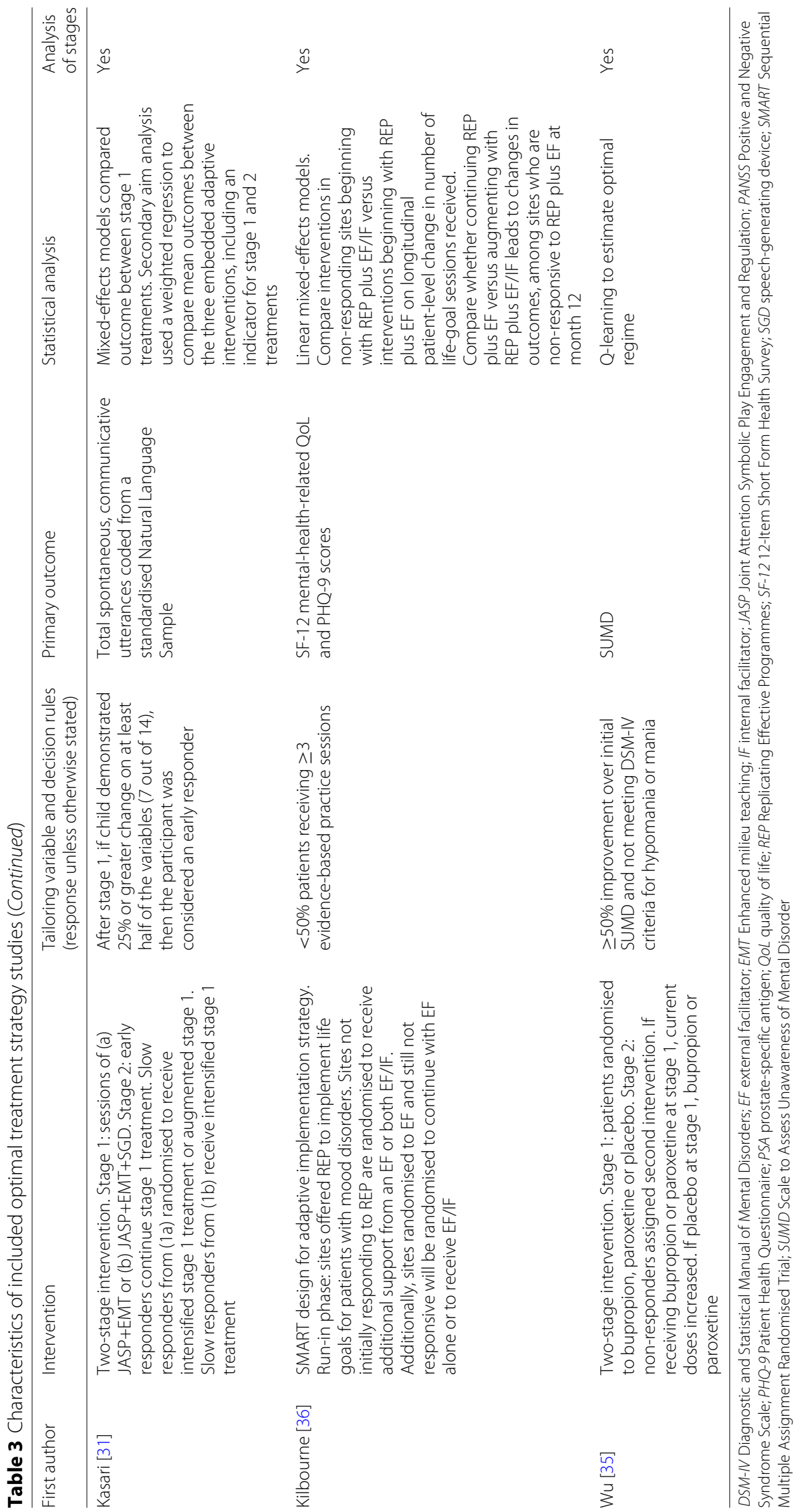




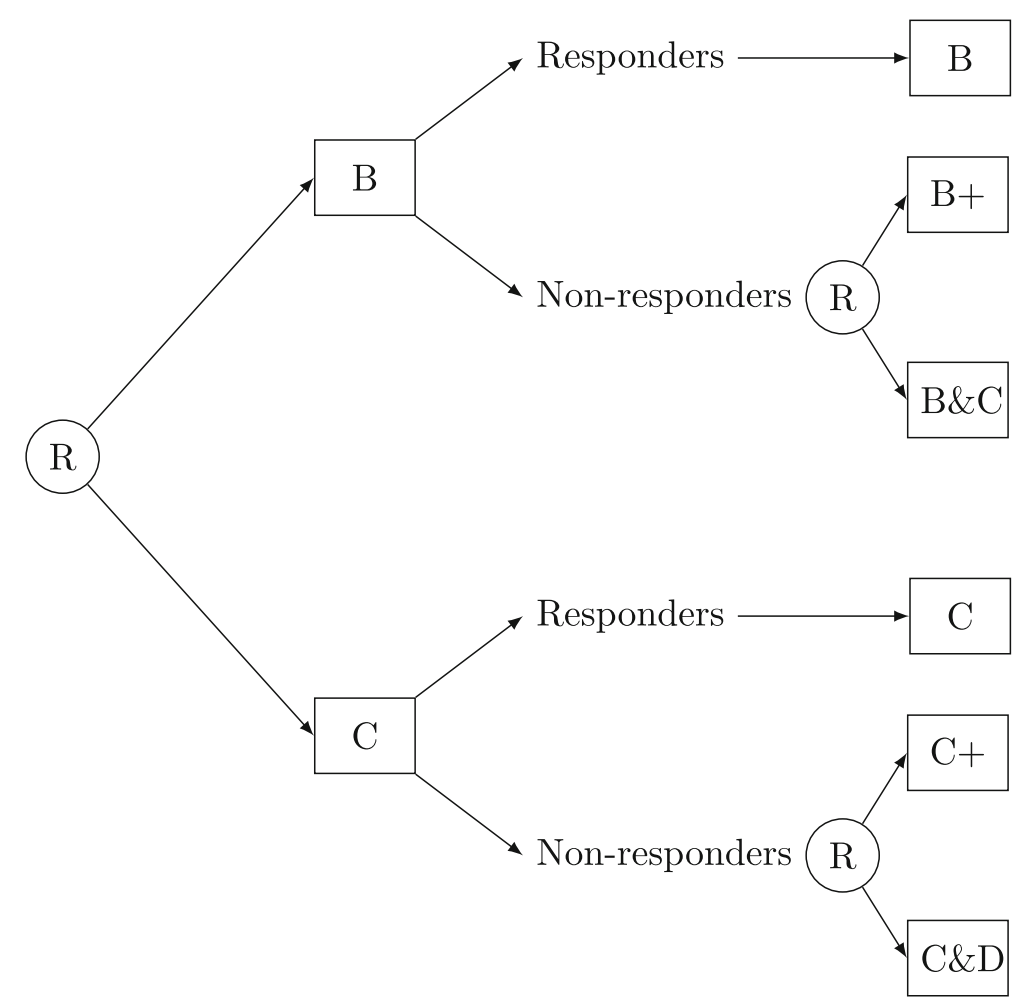

Fig. 4 SMART design with second randomisation dependent upon an intermediate outcome response status. SMART Sequential Multiple Assignment Randomised Trial, $R$ randomise, $B / B+, C / C+$ and $D$ represent different treatments, with for example, $B+$ being the more intense version of $B$

are not able to deal with missing data, (2) they cannot model the covariance among repeated measures and (3) a repeated measures ANOVA assumes there is an exchangeable auto-correlation structure between any two observations for the same individual [37]. More complex analysis methods were employed in the SMART studies that aimed to find the optimal adaptive treatment strategy.

Trials of proportionate interventions often lead to a complex hierarchical structure of data, with hierarchical clustering introduced by both treatment or centre, in addition to the longitudinal data.

A minority of studies considered the different stages of the interventions. Some stepped-care studies used an intention-to-treat analysis to compare the intervention group to the control group after each step individually and after the whole intervention period. Only one study explicitly evaluated the effectiveness of the different components as a key objective [17]. Without consideration of the separate component parts of a proportionate intervention we assume that each component will in itself be effective. Though this may be true, the effectiveness of the components might alter as they are incorporated with one another. By design, the population size of a stepped- care trial decreases as it passes through the steps. This makes any comparisons between stages either impossible or very difficult unless the study has been designed to account for this. It is possible to evaluate the effectiveness of each stage of a proportionate intervention, as done by Lamb [17], by randomising patients who are eligible to the active or control treatment, regardless of the treatment they received at the previous stage. In certain scenarios, it would be unethical or impossible to withhold the next stage treatment of a proportionate intervention if a patient were eligible (for example, if an unstaged version of the active treatment being tested was used as the control treatment or if each stage builds upon the previous stage).

\section{Limitations}

Due to resource limitations of this review, it was not possible to supplement the database by checking reference lists, conference proceedings or trial registries. Further work may include a supplementary search. We included only articles published in English. The studies included were mainly stepped care, which may suggest that the search criteria or eligibility criteria were unable to identify other types of studies that were also trials of proportionate 
interventions. We limited our review to articles published after 1 January 2010. However, this was to reflect current practice.

\section{Wider context}

There are increasing pressures on health and social care services, limited resources and increasing health inequalities. Proportionate interventions have a role to play in the overarching goal of proportionate universalism, both in reducing health inequalities and providing care to those in need. If early-stage low-intensity interventions provide similar outcomes to more intensive interventions, then costs can be reduced and the health interventions will be less onerous for some patients and for health professionals. Increasing the intensity of a treatment does not necessarily lead to increased effectiveness [38].

Additionally, proportionate interventions fit within the overarching goals of personalised medicine: to make decisions appropriate to an individual patient, to make decisions that lead to the best outcomes for the patient, and to formalise clinical decision-making and make it evidence based. Personalised medicine aims to assign individuals to interventions based on their individual characteristics and to target interventions to patients likely to benefit. This requires evidence on what types of patients will benefit from different interventions, which is not always available [39]. In contrast, proportionate interventions can be self-correcting, with individuals failing to benefit from lower intensity interventions stepping up to more intense interventions.

The recommendation from the Marmot review that interventions follow a proportionate universalism framework has not been supported by the evidence base on how to evaluate or implement such interventions [2]. The proportionate universalism framework has been discussed in NICE guidelines [40] and NHS documents [41-43] and by charities [44] and public health authorities [45]. However, little has been written in the academic literature on how to actually implement proportional universalism in practice or how to assess the effectiveness of these interventions. This review provides examples of the types of interventions that fit into the proportionate universalism framework and the trial designs used to evaluate these at present.

\section{Implications and recommendations}

There have been recent developments in adaptive treatment strategies, and trial designs now exist for optimal treatment strategies (SMARTs). There are also designs that evaluate the effectiveness of stepped-care treatments as a whole. Further research on how to design and analyse trials of proportionate interventions would benefit from considering when quantifying the effectiveness or the incremental effectiveness of each stage is necessary and how this may be implemented. This depends upon whether the separate stages have been evaluated in a trial before as well as the interactions between them. Is the interaction between the different components expected and of interest? Without this aspect, it may be unclear how all the components work and how they interact with one another.

Recent advances in designs of proportionate trials include just-in-time adaptive interventions. The design and framework are described in Nahum-Shani et al. [46] and Klasnja et al. [47]. This design is useful in, for example, the growing field of educational research for developing cluster-level adaptive interventions [48], or for comparing adaptive interventions embedded in a SMART [49]. Findings from trials using this framework are forthcoming and could form the basis of, or be included in, future systematic reviews on mobile health technologies.

Triallists need to account for the impact that multiple hierarchical levels (often present in proportionate interventions) have on the analysis. More complex mixedeffects models accounting for the various correlations may be necessary, including a consideration of methods for partially nested trials when clustering is present only in one arm [50,51].

Of the 51 studies excluded based on the full texts, 27 were excluded due to a lack of, or the undefined nature of, the decision stages or rules in the intervention. This lack of clarity was occasionally due to the decision rule being based on a health professional's opinion. However, a lack of clarity was also repeatedly due to limited information in the articles' explanation of what the intervention actually entailed. If a trial is to provide fully useable information and a replicable intervention, it must give a clear explanation of the decision stages and rules. The readers can then understand the reasoning, and the process can be implemented either in a different setting or in a further trial. When reporting trials, it is important to follow both the relevant Consolidated Standards of Reporting Trials (CONSORT) [52] and the Template for Intervention Description and Replication (TIDieR) [53]. Both state that interventions must be reported with sufficient detail to allow replication, including how and when they were administered. This is particularly pertinent in proportionate interventions, such as stepped care, since the how and when are often multifaceted.

\section{Conclusion}

The increasing demand on health and social care services and medicine has driven the move for proportionate universalism as well as the move towards fairer and more effective personalised medicine. Appropriate treatment and service provision according to individual need is key. Proportionate interventions aim to provide 
individuals with the care they require and reduce the burden of treatment on an individual whilst reserving resources for those most in need. The results of this review have identified various contexts and therapeutic areas in which trials of proportionate interventions are being designed and implemented, predominantly in the treatment of mental health disorders. The term 'proportionate universalism' was not used in any of the studies identified, though analogous terms were used, including the stepped-care model, adaptive treatment strategy and dynamic treatment regimen. The two key types of study designs found in this review included stepped-care studies and SMART studies. The effectiveness of the different stages was considered in a minority of studies and often only as a simple analysis using summary statistics. There is a need for a more consistent approach and further guidance on the design, analyses and reporting across trials of proportionate interventions, so that comparisons can be made.

\section{Additional files}

Additional file 1: Completed PRISMA 2009 checklist. (PDF 201 kb)

Additional file 2: Reference list of studies included in this systematic review. (PDF $46 \mathrm{~kb}$ )

\section{Abbreviations}

ANCOVA: Analysis of covariance; ANOVA: Analysis of variance; AUDIT-C: Alcohol use disorders identification test, consumption; BAl: Beck anxiety inventory; BSQ: Body sensations questionnaire; CBT: Cognitive behavioural therapy; CES-D: Epidemiologic studies depression scale; CGl: Clinical global impression; CGI-I: Clinical global impression, improvement scale; CGI-S: Clinical global impression, severity scale; CONSORT: Consolidated standards of reporting trials; COPD: Chronic obstructive pulmonary disease; CSR: Clinicians' severity rating; DSM-IV: Diagnostic and statistical manual of mental disorders; EF: External facilitator; EMT: Enhanced milieu teaching; EORTC-QLQ-C30: European Organisation for Research and Treatment of Cancer quality of life questionnaire; FAQ: Frequently asked questions; FBT: Family-based treatment; GAD-7: Generalised anxiety disorder 7; GP: General practitioner; HADS: Hospital anxiety and depression scale; HADS-A: Hospital anxiety and depression scale, anxiety; HADS-D: Hospital anxiety and depression scale, depression; ICD-10: International statistical classification of diseases and related health problems, 10th revision; IDS: Inventory of depressive symptomatology; IES: Impact of events scale; IF: Internal facilitator; IPC: Intensive parental coaching; JASP: Joint attention symbolic play engagement and regulation; K10: Kessler psychological distress scale; MASC: Multidimensional anxiety scale for children; MI: Motivational interview; MINI: Mini international neuropsychiatric interview; NHS: National health service; NICE: National institute for health and care excellence; NIHR: National institute for health research; PANSS: Positive and negative syndrome scale; PCTA: Parent-child treatment for anxiety; PHQ-9: Patient health questionnaire; PHQ-PD: Patient health questionnaire, panic disorder subscale; PHR: Public health research; PRISMA: Preferred reporting items for systematic reviews and meta-analyses; PSA: Prostate-specific antigen; PST: Problem-solving treatment; QoL: Quality of life; REP: Replicating effective programs; SCID-I: Structured clinical interview for DSM-IV; SCL-20: 20-item symptom checklist depression scale; SF-12: 12-item short form health survey; SGD: Speech-generating device; SMART: Sequential multiple assignment randomised trial; SPS: Sensory processing sensitivity; STAI: State-trait anxiety inventory; SUMD: Scale to assess unawareness of mental disorder; TIDieR: Template for intervention description and replication; TSCYC-PTS: Trauma symptom checklist for young children, post-traumatic stress subscale; WAD: Whiplash-associated disorders; WSAS: Work and social adjustment scale; Y-BOCS: Yale-brown obsessive-compulsive scale

\section{Acknowledgements}

We thank Professor Cindy Cooper for helpful comments on a draft of this article.

\section{Funding}

JCa is funded by the University of Sheffield Harry Worthington PhD Scholarship. MDT and JCo are funded by the University of Sheffield. TB is funded by the University of York. MDT, JCo and TB are partly funded by the National Institute for Health Research (NIHR) Public Health Research (PHR) Programme (project number 13/93/10).

Department of Health Disclaimer: The views and opinions expressed therein are those of the authors and do not necessarily reflect those of the NIHR PHR Programme, NIHR, NHS or the Department of Health.

\section{Availability of data and materials}

The data used during the current study are contained in Tables 1,2 and 3 of the manuscript. As this is a systematic review, all included papers have been published in full and are listed in the reference list and Additional file 1.

\section{Authors' contributions}

JCa drafted the study protocol, developed the search strategy, carried out the search, selected the studies and drafted the manuscript. MDT and JCO participated in its conception and design and checked a random sample of studies to confirm their suitability for inclusion. MDT, JCo and TB contributed to the search strategy and revised the paper critically. All authors read and approved the final manuscript.

Ethics approval and consent to participate

Not applicable.

\section{Consent for publication}

Not applicable.

\section{Competing interests}

The authors declare that they have no competing interests.

\section{Publisher's Note}

Springer Nature remains neutral with regard to jurisdictional claims in published maps and institutional affiliations.

\section{Author details}

${ }^{1}$ ScHARR, University of Sheffield, 30 Regent Court, S1 4DA Sheffield, UK ${ }^{2}$ Department of Health Sciences, University of York, Area 2, Seebohm, Rowntree Building, Y010 5DD York, UK. ${ }^{3}$ Hull Health Trials Unit, University of Hull/Hull York Medical School, York, UK.

Received: 14 June 2017 Accepted: 17 January 2019

Published online: 28 February 2019

\section{References}

1. Almirall D, Kasari C, McCaffrey DF, Nahum-Shani I. Developing optimized adaptive interventions in education. J Res Educ Eff. 2018;11(1):27-34.

2. Marmot MG, Allen J, Goldblatt P, Boyce T, McNeish D, Grady M, Geddes I, et al. Fair society, healthy lives: Strategic review of health inequalities in England post-2010. The Marmot Review. 2010.

3. Nahum-Shani I, Qian M, Almirall D, Pelham WE, Gnagy B, Fabiano GA, Waxmonsky JG, Yu J, Murphy SA. Experimental design and primary data analysis methods for comparing adaptive interventions. Psychol Methods. 2012;17(4):457.

4. Medical Research Council. Developing and evaluating complex interventions: new guidance. https://www.mrc.ac.uk/documents/pdf/ complex-interventions-guidance. Accessed 14 May 2017.

5. Lancaster G, Campbell M, Eldridge S, Farrin A, Marchant M, Muller S, Perera R, Peters T, Prevost A, Rait G. Trials in primary care: statistical issues in the design, conduct and evaluation of complex interventions. Stat Methods Med Res. 2010;19(4):349-77.

6. Collins LM, Murphy SA, Bierman KL. A conceptual framework for adaptive preventive interventions. Prev Sci. 2004;5(3):185-96.

7. Moher D, Liberati A, Tetzlaff J, Altman DG, Group P, et al. Preferred reporting items for systematic reviews and meta-analyses: the PRISMA statement. PLoS Med. 2009;6(7):1000097. 
8. Cohcrane Collaboration. Cochrane Highly Sensitive Search Strategy for identifying randomized trials in MEDLINE: sensitivity- and precision-maximizing version (2008 revision); Ovid format. http://handbook.cochrane.org/chapter_6/box_6.4.d_cochrane_hsss_ sensprec_ovid.htm.

9. National Institute for Health and Care Excellence. Common mental health disorders: Identification and pathways to care. NICE guidelines [CG123]. 2011. https://www.nice.org.uk/guidance/CG123.

10. Paris J. Stepped care: an alternative to routine extended treatment for patients with borderline personality disorder. Psychiatr Serv. 2013;64(10): 1035-7.

11. Jakicic JM, Tate DF, Lang W, Davis KK, Polzien K, Rickman AD, Erickson K, Neiberg RH, Finkelstein EA. Effect of a stepped-care intervention approach on weight loss in adults: a randomized clinical trial. JAMA 2012;307(24):2617-26.

12. Carels RA, Hoffmann DA, Hinman N, Burmeister JM, Koball A, Ashrafioun L, Oehlhof MW, Bannon E, Leroy M, Darby L. Step-down approach to behavioural weight loss treatment: a pilot of a randomised clinical trial. Psychol Health. 2013;28(10):1121-34

13. Watson J, Crosby H, Dale V, Tober G, Wu Q, Lang J, McGovern R, Newbury-Birch D, Parrott S, Bland J, et al. AESOPS: a randomised controlled trial of the clinical effectiveness and cost-effectiveness of opportunistic screening and stepped care interventions for older hazardous alcohol users in primary care. Health Technology Assessment, No. 17.25. 2013

14. Borsari B, Magill M, Mastroleo NR, Hustad JTP, Tevyaw TOL, Barnett NP, Kahler CW, Eaton E, Monti PM. Mandated college students' response to sequentially administered alcohol interventions in a randomized clinical trial using stepped care. J Consult Clin Psychol. 2016;84(2):103-12.

15. Lock J, Le Grange D, Agras WS, Fitzpatrick KK, Jo B, Accurso E, Forsberg S, Anderson K, Arnow K, Stainer M. Can adaptive treatment improve outcomes in family-based therapy for adolescents with anorexia nervosa? Feasibility and treatment effects of a multi-site treatment study. Behav Res Ther. 2015;73:90-5.

16. Mitchell JE, Agras S, Crow S, Halmi K, Fairburn CG, Bryson S, Kraemer H. Stepped care and cognitive-behavioural therapy for bulimia nervosa: randomised trial. Br J Psychiatry. 2011;198(5):391-7.

17. Lamb SE, Williams MA, Williamson EM, Gates S, Withers EJ, Mt-Isa S, Ashby D, Castelnuovo E, Underwood M, Cooke MW, Group MT. Managing Injuries of the Neck Trial (MINT): a randomised controlled trial of treatments for whiplash injuries. Health Technol Assess. 2012;16(49).

18. Richter D, Mickel C, Acharya S, Brunel P, Militaru C. Aliskiren-based stepped-care treatment algorithm provides effective blood pressure control. Int J Clin Pract. 2011;65(5):613-23.

19. Hamall KM, Heard TR, Inder KJ, McGill KM, Kay-Lambkin F. The Child Illness and Resilience Program (CHiRP): a study protocol of a stepped care intervention to improve the resilience and wellbeing of families living with childhood chronic illness. BMC Psychol. 2014;2(1).

20. Stam H, van der Wouden JC, van der Horst HE, Maarsingh OR. Impairment reduction in older dizzy people in primary care: study protocol for a cluster randomised controlled trial. Trials. 2015;16(313).

21. Borsari B, Hustad JTP, Mastroleo NR, Tevyaw TO, Barnett NP, Kahler CW, Short EE, Monti PM. Addressing alcohol use and problems in mandated college students: a randomized clinical trial using stepped care. J Consult Clin Psychol. 2012;80(6):1062-74.

22. Mitchell N, Hewitt C, Adamson J, Parrott S, Torgerson D, Ekers D, Holmes J, Lester H, McMillan D, Richards D, Spilsbury K, Godfrey C, Gilbody S. A randomised evaluation of CollAborative care and active surveillance for Screen-Positive EldeRs with sub-threshold depression (CASPER): study protocol for a randomized controlled trial. Trials. 2011;12.

23. Tolin DF, Diefenbach GJ, Gilliam CM. Stepped care versus standard cognitive-behavioral therapy for obsessive-compulsive disorder: a preliminary study of efficacy and costs. Depression Anxiety. 2011;28(4): 314-23.

24. Nordin K, Rissanen R, Ahlgren J, Burell G, Fjällskog ML, Börjesson S, Arving C. Design of the study: how can health care help female breast cancer patients reduce their stress symptoms? A randomized intervention study with stepped-care. BMC Cancer. 2012;12(1):167.

25. Gilliam CM, Diefenbach GJ, Whiting SE, Tolin DF. Stepped care for obsessive-compulsive disorder: an open trial. Behav Res Ther. 2010;48(11):1144-9.
26. van der Leeden AJ, van Widenfelt BM, van der Leeden R, Liber JM, Utens EM, Treffers PD. Stepped care cognitive behavioural therapy for children with anxiety disorders: a new treatment approach. Behav Cogn Psychother. 2011;39(1):55-75.

27. Krebber $A M H$, Leemans $C R$, de Bree $R$, van Straten A, Smit F, Smit EF, Becker A, Eeckhout GM, Beekman AT, Cuijpers P, et al. Stepped care targeting psychological distress in head and neck and lung cancer patients: a randomized clinical trial. BMC Cancer. 2012;12(1):173.

28. Mattsson S, Alfonsson S, Carlsson M, Nygren P, Olsson E, Johansson B. U-CARE: internet-based stepped care with interactive support and cognitive behavioral therapy for reduction of anxiety and depressive symptoms in cancer: a clinical trial protocol. BMC Cancer. 2013;13:414.

29. Oosterbaan DB, Verbraak MJ, Terluin B, Hoogendoorn AW, Peyrot WJ, Muntingh A, van Balkom AJ. Collaborative stepped care versus care as usual for common mental disorders: 8-month, cluster randomised controlled trial. Br J Psychiatry. 2013;203(2):132-9.

30. Rose JE, Behm FM. Adapting smoking cessation treatment according to initial response to precessation nicotine patch. Am J Psychiatry. 2013;170(8):860-7.

31. Kasari C, Kaiser A, Goods K, Nietfeld J, Mathy P, Landa R, Murphy S, Almirall D. Communication interventions for minimally verbal children with autism: a sequential multiple assignment randomized trial. J Am Acad Child Adolesc Psychiatry. 2014;53(6):635-46.

32. Weiss RD, Potter JS, Fiellin DA, Byrne M, Connery HS, Dickinson W, Gardin J, Griffin ML, Gourevitch MN, Haller DL, et al. Adjunctive counseling during brief and extended buprenorphine-naloxone treatment for prescription opioid dependence: a 2-phase randomized controlled trial. Arch Gen Psychiatr. 2011;68(12):1238-46.

33. Shortreed SM, Moodie EE. Estimating the optimal dynamic antipsychotic treatment regime: evidence from the sequential multiple-assignment randomized clinical antipsychotic trials of intervention and effectiveness schizophrenia study. J R Stat Soc Ser C Appl Stat. 2012;61(4):577-99.

34. Wang L, Rotnitzky A, Lin X, Millikan RE, Thall PF. Evaluation of viable dynamic treatment regimes in a sequentially randomized trial of advanced prostate cancer. J Am Stat Assoc. 2012;107(498):493-508.

35. Wu F, Laber EB, Lipkovich IA, Severus E. Who will benefit from antidepressants in the acute treatment of bipolar depression? A reanalysis of the STEP-BD study by Sachs et al. 2007, using Q-learning. Int J Bipolar Disord. 2015;3(1):1-11.

36. Kilbourne AM, Almirall D, Eisenberg D, Waxmonsky J, Goodrich DE, Fortney JC, Kirchner JE, Solberg LI, Main D, Bauer MS. Protocol: Adaptive Implementation of Effective Programs Trial (ADEPT): cluster randomized SMART trial comparing a standard versus enhanced implementation strategy to improve outcomes of a mood disorders program. Implement Sci. 2014;9(132):

37. Diggle P, Heagerty P, Liang KY, Zeger S. Analysis of Longitudinal Data. Oxford: Oxford University Press; 2002.

38. Andersson G, Carlbring P, Draper C, O'Donohue W. Stepped Care and E-health: Practical Applications to Behavioral Disorders. New York: Springer; 2011.

39. Richards DA, Bower P, Pagel C, Weaver A, Utley M, Cape J, Pilling S, Lovell K, Gilbody S, Leibowitz J, et al. Delivering stepped care: an analysis of implementation in routine practice. Implementation Sci. 2012;7(1):1.

40. NICE. Health inequalities and population health. NICE advice [LGB4]. 2012 https://www.nice.org.uk/advice/lgb4.

41. NHS Scotland. Proportionate Universalism Briefing. 2014. http://www. healthscotland.com/uploads/documents/24296ProportionateUniversalismBriefing.pdf. Accessed 29 Apr 2016.

42. Healthy Children. Transforming Child Health Information. National Information Board, NHS England. NHS England Publications Gateway Reference 05454. https://www.england.nhs.uk/digitaltechnology/childhealth/. Accessed 2 Dec 2016.

43. Inequalities in the early years. NHS Scotland. http://www.maternal-andearly-years.org.uk/topic/3-5-years/inequalities-in-the-early-years. Accessed 29 Apr 2016.

44. Hutt $P$, Gilmore S. Tackling inequalities in general practice. The Kings Fund. 2010. https://www.kingsfund.org.uk/sites/files/kf/Health. Accessed 29 Apr 2016.

45. Lu D, Tyler I. Focus On: A Proportionate Approach to Priority Populations. Public Health Ontario. https://www.publichealthontario.ca/en/ eRepository/Focus_On_Priority_Populations.pdf. Accessed 29 Apr 2016. 
46. Nahum-Shani I, Hekler EB, Spruijt-Metz D. Building health behavior models to guide the development of just-in-time adaptive interventions: a pragmatic framework. Health Psychol. 2015;34(S):1209.

47. Klasnja P, Hekler EB, Shiffman S, Boruvka A, Almirall D, Tewari A, Murphy SA. Microrandomized trials: an experimental design for developing just-in-time adaptive interventions. Health Psychol. 2015;34(S):1220.

48. NeCamp T, Kilbourne A, Almirall D. Comparing cluster-level dynamic treatment regimens using sequential, multiple assignment, randomized trials: regression estimation and sample size considerations. Stat Methods Med Res. 2017;26(4):1572-89.

49. LuX, Nahum-Shani I, Kasari C, Lynch KG, Oslin DW, Pelham WE, Fabiano G, Almirall D. Comparing dynamic treatment regimes using repeated-measures outcomes: modeling considerations in smart studies. Stat Med. 2016:35(10):1595-615.

50. Roberts C, Roberts SA. Design and analysis of clinical trials with clustering effects due to treatment. Clin Trials. 2005;2(2):152-62.

51. Baldwin SA, Bauer DJ, Stice E, Rohde P. Evaluating models for partially clustered designs. Psychol Methods. 2011;16(2):149-165.

52. Schulz KF, Altman DG, Moher D. CONSORT 2010 statement: updated guidelines for reporting parallel group randomised trials. BMC Med. 2010;8(1):1.

53. Hoffmann TC, Glasziou PP, Boutron I, Milne R, Perera R, Moher D, Altman DG, Barbour V, Macdonald $H$, Johnston $M$, et al. Better reporting of interventions: template for intervention description and replication (TIDieR): checklist and guide. BMJ. 2014;348:1687.

54. Ell K, Katon W, Xie B, Lee PJ, Kapetanovic S, Guterman J, Chou CP. Collaborative care management of major depression among low-income, predominantly Hispanic subjects with diabetes: a randomized controlled trial. Diabetes Care. 2010;33(4):706-13.

55. van't Veer-Tazelaar $P$, Smit $F$, van Hout $H$, van Oppen $P$, van der Horst $H$, Beekman A, van Marwijk H. Cost-effectiveness of a stepped care intervention to prevent depression and anxiety in late life: randomised trial. Br J Psychiatry. 2010;196(4):319-25.

56. Braamse AMJ, van Meijel $B$, Visser $O$, van Oppen $P$, Boenink AD, Eeltink $C$, Cuijpers P, Huijgens PC, Beekman ATF, Dekker J. Distress and quality of life after autologous stem cell transplantation: a randomized clinical trial to evaluate the outcome of a web-based stepped care intervention. BMC Cancer. 2010;10(361).

57. Patel V, Weiss HA, Chowdhary N, Naik S, Pednekar S, Chatterjee S, De Silva MJ, Bhat B, Araya R, King M, et al. Effectiveness of an intervention led by lay health counsellors for depressive and anxiety disorders in primary care in Goa, India (MANAS): a cluster randomised controlled trial. Lancet. 2010;376(9758):2086-95.

58. Kay-Lambkin FJ, Baker AL, McKetin R, Lee N. Stepping through treatment: reflections on an adaptive treatment strategy among methamphetamine users with depression. Drug Alcohol Rev. 2010;29(5):475-82.

59. Seekles W, van Straten A, Beekman A, van Marwijk H, Cuijpers $P$. Stepped care treatment for depression and anxiety in primary care: a randomized controlled trial. Trials. 2011;12(171)

60. Apil SR, Hoencamp E, Haffmans JPM, Spinhoven P. A stepped care relapse prevention program for depression in older people: a randomized controlled trial. Int J Geriatr Psychiatry. 2012;27(6):583-91.

61. Karp JF, Rollman BL, Reynolds CF, Morse JQ, Lotrich F, Mazumdar S, Morone N, Weiner DK. Addressing both depression and pain in late life: the methodology of the ADAPT study. Pain Med. 2012;13(3):405-18.

62. Dozeman E, van Marwijk HW, van Schaik DJ, Smit F, Stek ML, van der Horst HE, Bohlmeijer ET, Beekman AT. Contradictory effects for prevention of depression and anxiety in residents in homes for the elderly: a pragmatic randomized controlled trial. Int Psychogeriatr. 2012;24(08):1242-51.

63. Pommer AM, Pouwer F, Denollet J, Pop VJ. Managing co-morbid depression and anxiety in primary care patients with asthma and/or chronic obstructive pulmonary disease: study protocol for a randomized controlled trial. Trials. 2012;13(1):6

64. Van Dijk SE, Pols AD, Adriaanse MC, Bosmans JE, Elders PJ, Van Marwijk HW, Van Tulder MW. Cost-effectiveness of a stepped-care intervention to prevent major depression in patients with type 2 diabetes mellitus and/or coronary heart disease and subthreshold depression: design of a cluster-randomized controlled trial. BMC Psychiatry. 2013;13(128).

65. Arving C, Thormodsen I, Brekke G, Mella O, Berntsen S, Nordin K. Early rehabilitation of cancer patients: a randomized controlled intervention study. BMC Cancer. 2013;13.
66. van der Aa HP, Van Rens GH, Comijs HC, Bosmans JE, Margrain TH, van Nispen RM. Stepped-care to prevent depression and anxiety in visually impaired older adults: design of a randomised controlled trial. BMC Psychiatry. 2013;13(209).

67. Muntingh A, van der Feltz-Cornelis $C$, van Marwijk H, Spinhoven $P$, Assendelft W, de Waal M, Ader H, van Balkom A. Effectiveness of collaborative stepped care for anxiety disorders in primary care: a pragmatic cluster randomised controlled trial. Psychother Psychosom. 2014;83(1):37-44.

68. Gureje O, Oladeji BD, Araya R, Montgomery AA. A cluster randomized clinical trial of a stepped care intervention for depression in primary care (STEPCARE): study protocol. BMC Psychiatry. 2015;15(1):1.

69. Stoop C, Nefs G, Pommer A, Pop V, Pouwer F. Effectiveness of a stepped care intervention for anxiety and depression in people with diabetes, asthma or COPD in primary care: a randomized controlled trial. J Affect Disord. 2015;184:269-76.

70. Schuurhuizen CS, Braamse AM, Beekman AT, Bomhof-Roordink H, Bosmans JE, Cuijpers P, Hoogendoorn AW, Konings IR, van der Linden $\mathrm{MH}$, Neefjes EC, et al. Screening and treatment of psychological distress in patients with metastatic colorectal cancer: study protocol of the TES trial. BMC Cancer. 2015;15(302).

71. Haug T, Nordgreen T, Öst LG, Kvale G, Tangen T, Andersson G, Carlbring P, Heiervang ER, Havik OE. Stepped care versus face-to-face cognitive behavior therapy for panic disorder and social anxiety disorder: predictors and moderators of outcome. Behav Res Ther. 2015;71:76-89.

72. Salloum A, Wang W, Robst J, Murphy TK, Scheeringa MS, Cohen JA, Storch EA. Stepped care versus standard trauma-focused cognitive behavioral therapy for young children. J Child Psychol Psychiatry. 2015;57(5):614-22.

73. Painter JT, Fortney JC, Gifford AL, Rimland D, Monson T, RodriguezBarradas MC, Pyne JM. Cost-effectiveness of collaborative care for depression in HIV clinics. J Acquir Immune Defic Syndr. 2015;70(4):377-85.

\section{Ready to submit your research? Choose BMC and benefit from:}

- fast, convenient online submission

- thorough peer review by experienced researchers in your field

- rapid publication on acceptance

- support for research data, including large and complex data types

- gold Open Access which fosters wider collaboration and increased citations

- maximum visibility for your research: over $100 \mathrm{M}$ website views per yea

At BMC, research is always in progress.

Learn more biomedcentral.com/submissions 